\title{
Time-Frequency Representation of Signals Using Kalman Filter
}

\author{
Jindřich Liška and Eduard Janeček \\ Department of Cybernetics, University of West Bohemia in Pilsen, \\ Univerzitní 8, 30614 Pilsen, \\ Czech Republic
}

\section{Introduction}

Data analysis is a necessary part of practical applications as well as of pure research. The problem of data analysis is of great interest in engineering areas such as signal processing, speech recognition, vibration analysis, time series modelling, etc. Sensing of physical effects transformed to time series of voltage represents measured reality for us. However, the knowledge of time characteristics of measured data is often quite insufficient for description of real signal properties. Therefore, frequency analysis, as an alternative signal description approach, has been engaged in signal processing methods. Unfortunately, data from real applications are mostly non-stationary and represent non-linear processes in general. This fact has leaded to introduction of joint time-frequency representation of measured signals.

Former time-frequency methods dealing with non-stationary data analysis, such as the short-time Fourier transform, repeatedly use a block of data processing with the assumption that frequency characteristics are time-invariant and/or a process is stationary within the usage of the data block. The resolution of such methods is then given by the HeisenbergGabor uncertainty principle. This limitation is mainly addressed by Fourier pairs and by the block processing.

In this chapter, the definition of instantaneous frequency introduces a new approach to acquire a proper time-frequency representation of a signal. The description of limitations which have to be taken into account in order to achieve meaningful results is also given there.

The section that deals with the instantaneous frequency phenomenon is followed by the part describing the construction of the signal model. A signal is modelled as an output of a system that consists of sum of auto-regressive (AR), linear time-invariant (LTI), secondorder subsystems. In fact, such a model corresponds to a system of resonators formed in parallel. The reason for such model selection is simplicity in matrix implementation as well as preservation of a physical meaning of decomposed signal components. The signal model is developed in state space and there is also demonstrated how to select adequately all system matrices.

The estimation of particular signal components is then obtained through the adaptive Kalman filtering which is based on the previously defined state space model. The Kalman filter recursively estimates time-varying signal components in a complex form. The complex form is required in order to compute the instantaneous frequency and amplitude of the 
signal components. Furthermore, the algorithm recursive form facilitates implementations in signal processing devices. Initial parameters of the Kalman filter are obtained from frequency spectrum characteristics and from estimation of signal spectral density through the adaptive algorithm by matching the auto-regressive prediction model and the signal.

To illustrate performance of the proposed method, experimental results are presented in order to show comparisons of common time-frequency techniques and the Kalman filter method. Moreover, results from analysis of example signals (chirp signal, step frequency change, etc.) are added. The contribution of this method mainly consists in improvement of the time-frequency resolution as discussed in the last section, which concludes the whole chapter.

\section{Instantaneous frequency and the complex signal}

In mechanics, the frequency of vibration motion is defined as the number of oscillations per time period. In the course of one oscillation, the body deflects from the equilibrium, goes through the extremes and the oscillation ends again in the equilibrium position. The simple harmonic sine wave is often used for representation of such a vibration motion. The harmonic motion actually represents projection of circle body move with uniform velocity on a circle diameter.

However, in many applications the motion velocity and therefore also the oscillation frequency changes in time. Signals with these properties are often referred to as nonstationary and their important characteristic is the dependence of frequency on time, therefore the notion of instantaneous frequency. In other words, instantaneous frequency is the time-varying parameter which describes the location of the signal's spectral peak in time. It can be interpreted as the frequency of a sine wave which locally fits the analyzed signal, but physically it has meaning only for monocomponent signals, where there is only one frequency or a narrow range of frequencies varying as a function of time (Boashash, 1992a).

Let's assume the simple harmonic motion in following form:

$$
s_{r}(t)=a \cos (\omega t+\theta), \quad \omega=2 \pi f
$$

where $a$ is amplitude, $\omega$ is the angular frequency, $\theta$ is a phase constant and the argument of the cosine function is the instantaneous phase $\phi(t)$ (namely $\omega t+\theta$ ). In the case of frequency changing the instantaneous phase $\phi(t)$ is then the integral of frequency in time and the signal form should be rewritten as

$$
s_{r}(t)=a \cos \left(\int_{0}^{t} \omega(t) d t+\theta\right)
$$

Considering the monocomponent signal, instantaneous frequency $\omega(t)$ is defined, with respect to (2), as a derivation of phase $\phi(t)$

$$
\omega(t)=\frac{d \phi(t)}{d t}=2 \pi f(t)
$$

In most cases, there is no way how to determine the instantaneous phase directly from the real signal. One of the tricks how to obtain the unknown phase is the introduction of 
complex signal $z(t)$ which somehow corresponds to the real signal. As described in (Hahn, 1996) or in (Huang, 1998), the Hilbert transform is the elegant solution for generating of complex signal from the real one. The Hilbert transform of a real signal $s_{r}(t)$ of the continuous variable $t$ is

$$
s_{i}(t)=\frac{1}{\pi} P \int_{-\infty}^{\infty} \frac{s_{r}(\eta)}{\eta-t} d \eta
$$

where $P$ indicates the Cauchy Principle Value integral. The complex signal $z(t)$

$$
z(t)=s_{r}(t)+j \cdot s_{i}(t)=a(t) e^{j \varphi(t)},
$$

whose imaginary part is the Hilbert transform $s_{i}(t)$ of the real part $s_{r}(t)$ is then called the analytical signal and its spectrum is composed only of the positive frequencies of the real signal $s_{r}(t)$.

From the complex signal, an instantaneous frequency and amplitude can be obtained for every value of $t$. Following (Hahn, 1996) the instantaneous amplitude is simply defined as

$$
a(t)=\sqrt{s_{r}(t)^{2}+s_{i}(t)^{2}}
$$

and similarly the instantaneous phase as

$$
\varphi(t)=\arctan \frac{s_{i}(t)}{s_{r}(t)} .
$$

The instantaneous frequency is then equal to

$$
\omega(t)=\frac{d}{d t}\left(\arctan \frac{s_{i}(t)}{s_{r}(t)}\right)=\frac{s_{r}(t) \dot{s}_{i}(t)-s_{i}(t) \dot{s}_{r}(t)}{s_{r}(t)^{2}+s_{i}(t)^{2}} .
$$

Even with the Hilbert transform, there is still considerable controversy in defining the instantaneous frequency as mentioned also in (Boashash, 1992a). Applying the Hilbert transform directly to a multicomponent signal provides values of $a(t)$ and $\omega(t)$ which are unusable for describing the signal. The idea of instantaneous frequency and amplitude does not make sense when a signal consists of multiple components at different frequencies. It leads (Huang, 1998) to introduce a so called Empirical Mode Decomposition method to decompose the signal into monocomponent functions (Intrinsic Mode Functions). In this work, another method for signal decomposition is introduced.

\section{Complex signal component model}

Let's consider the multicomponent real signal $s_{r}(t)$

$$
s_{r}(t)=\sum_{n=1}^{N} s_{r}^{(n)}(t)+\rho(t)
$$

which consists from noise $\rho(t)$ representing any undesirable components and from $N$ single component nonstationary signals described by envelopes $a$ and frequencies $\omega$. 


$$
s_{r}^{(n)}(t)=a_{n} \cdot \cos \left(\omega_{n} \cdot t\right)
$$

The first derivation of the signal component is

$$
\dot{s}_{r}^{(n)}(t)=-a_{n} \cdot \omega_{n} \cdot \sin \left(\omega_{n} \cdot t\right)
$$

and the second one is described as follows

$$
\ddot{s}_{r}^{(n)}(t)=-a_{n} \cdot \omega_{n}^{2} \cos \left(\omega_{n} \cdot t\right)=-\omega_{n}^{2} \cdot s_{r}^{(n)}(t)
$$

On the basis of equation (12) the state space model of signal component $s_{r}^{(n)}(t)$ can be derived as second-order $(n=2)$ model of auto-regressive (AR), linear time-invariant (LTI) system.

Let's assume an AR state space model with state $x(t)$

$$
\dot{x}(t)=A \cdot x(t)
$$

and system's model output $y(t)$

$$
y(t)=C \cdot x(t)
$$

where $A$ is the state matrix and $C$ is the output matrix. The state vector $x(t)$ consists, in our case, of two internal states: real state part $x_{r}(t)$ and imaginary part $x_{i}(t)$.

With respect to the former introduced notation of the signal components and considering the analytical signal, we can choose the state vector components as $x_{r}^{(n)}=\cos \left(\omega_{n} t\right)$ and $x_{i}^{(n)}=\sin \left(\omega_{n} t\right)$. This choice takes into account the Hilbert transform and the orthogonal character of both components. Hence, the corresponding state space model is represented by the following state equation

$$
\left[\begin{array}{c}
\dot{x}_{r}^{(n)}(t) \\
\dot{x}_{i}^{(n)}(t)
\end{array}\right]=\left[\begin{array}{cc}
0 & -\omega_{n} \\
\omega_{n} & 0
\end{array}\right] \cdot\left[\begin{array}{l}
x_{r}^{(n)}(t) \\
x_{i}^{(n)}(t)
\end{array}\right]
$$

and the model's output is

$$
y_{n}(t)=\left[\begin{array}{ll}
1 & 0
\end{array}\right] \cdot\left[\begin{array}{l}
x_{r}^{(n)}(t) \\
x_{i}^{(n)}(t)
\end{array}\right] .
$$

In this case the model output $y_{n}(t)$ represents the signal component $s_{r}^{(n)}(t)$. The state matrix $A$ of the model is a $2 \mathrm{D}$ rotation matrix whose eigenvalues are pure imaginary numbers. The trajectory in state space is a circle and the model represents in fact an undamped resonator (oscillator) with natural frequency in $\omega_{n}$. The solution of the state equation consist only from the homogenous part (there is no model input) and is described by the following form

$$
x_{r}^{(n)}(t)=e^{A\left(t-t_{0}\right)} x_{r}^{(n)}\left(t_{0}\right) .
$$

Computing state transition matrix $e^{A t}$ and using discretization step of $\Delta t=h$, the discrete state space representation is acquired as 


$$
\left[\begin{array}{l}
x_{r}^{(n)}(k+1) \\
x_{i}^{(n)}(k+1)
\end{array}\right]=\left[\begin{array}{cc}
\cos \left(h \cdot \omega_{n}\right) & -\sin \left(h \cdot \omega_{n}\right) \\
\sin \left(h \cdot \omega_{n}\right) & \cos \left(h \cdot \omega_{n}\right)
\end{array}\right] \cdot\left[\begin{array}{l}
x_{r}^{(n)}(k) \\
x_{i}^{(n)}(k)
\end{array}\right]+\Gamma(k) \cdot \xi(k) .
$$

and the output equation is

$$
y_{n}(k)=\left[\begin{array}{ll}
1 & 0
\end{array}\right] \cdot\left[\begin{array}{c}
x_{r}^{(n)}(k) \\
x_{i}^{(n)}(k)
\end{array}\right] .
$$

The generalized output equation for all signal components is then

$$
y(k)=C \cdot x(k)+\Delta \cdot \eta(k) .
$$

In previous equations, $\xi(k)$ is the state noise and $\eta(k)$ is the output noise of state model. Both noise vectors, $\xi(k)$ and $\eta(k)$, are zero-centred with identity covariance matrices. The specific features of the noises are characterized by the covariance matrix $\Gamma$ and value $\Delta$. This derived resonator model forms together with Kalman filtering approach an estimator of analytical signal. The estimation of the first model state is a real part (sine function) of the signal component and estimation of the second state is an imaginary part (cosine function).

\subsection{Selection of matrices $\Gamma$ and value $\Delta$}

The choice of the proper matrix $\Gamma$ and value $\Delta$ is the important part in definition of the signal component model. These two parameters decide what amount of energy of the original signal will be assigned to the actual signal component.

We have already defined the description of the complex signal in equation (5) which represents the general output of the component model. Let's assume that the amplitude $a$ of the $\mathrm{n}^{\text {th }}$ complex signal component $z_{n}(k)$ is not constant but it changes from sample $k$ to sample $k+1$ of deviation $\varepsilon(k)$ :

$$
z_{n}(k+1)=(a(k)+\varepsilon(k)) \cdot e^{j \varphi(k+1)} .
$$

It means that the amplitude in sample $k+1$ is described in recursive form by the following equation

$$
a(k+1)=a(k)+\varepsilon(k), \text { where } \varepsilon(k) \sim N\left(0, \sigma_{\varepsilon}{ }^{2}\right) .
$$

The time changing phasor with its corresponding amplitude $a(k)$, phase $\varphi(k)$ and deviation $\varepsilon(k)$ is displayed in Fig.1. The real trajectory of the complex signal mode is marked by black curve and phasor trajectory in case of the constant amplitude in time $k$ is signed as greydashed circle. The recursive form of the amplitude progression model is shown in the left picture in Fig. 1. Each phasor amplitude differs from the previous one (green dotted line) of deviation $\varepsilon$ (red line).

The right picture of the Fig. 1. shows in detail the progression of the phasor in samples $k$ and $k+1$. The characteristics of the $\gamma_{1}$ and $\gamma_{2}$ parameters are derived in the following text.

The state in the sample $k+1$ in dependence on the state in sample $k$ is described, as above defined, in the following form

$$
x(k+1)=A \cdot x(k)+\Gamma(k) \cdot \xi(k), \text { where } \xi(k) \sim N(0,1)
$$


Equation (23) describes model, where the complex signal $x(k)$ is firstly rotated by the matrix $A$ and the sum with $\varepsilon(k)$ produces a new state vector $x(k+1)$. Let's assume equation (23) in a new form, where vector $x(k)$ is firstly summed with $\varepsilon(k)$ and as late as then rotated by matrix $A$ :

$$
x(k+1)=A \cdot(x(k)+\bar{\Gamma}(k) \cdot \xi(k))
$$

This form is used in order to reveal the relation between $\varepsilon$ and $\Gamma$ and that is why the new vector $\bar{\Gamma}$ is introduced into the state equation.
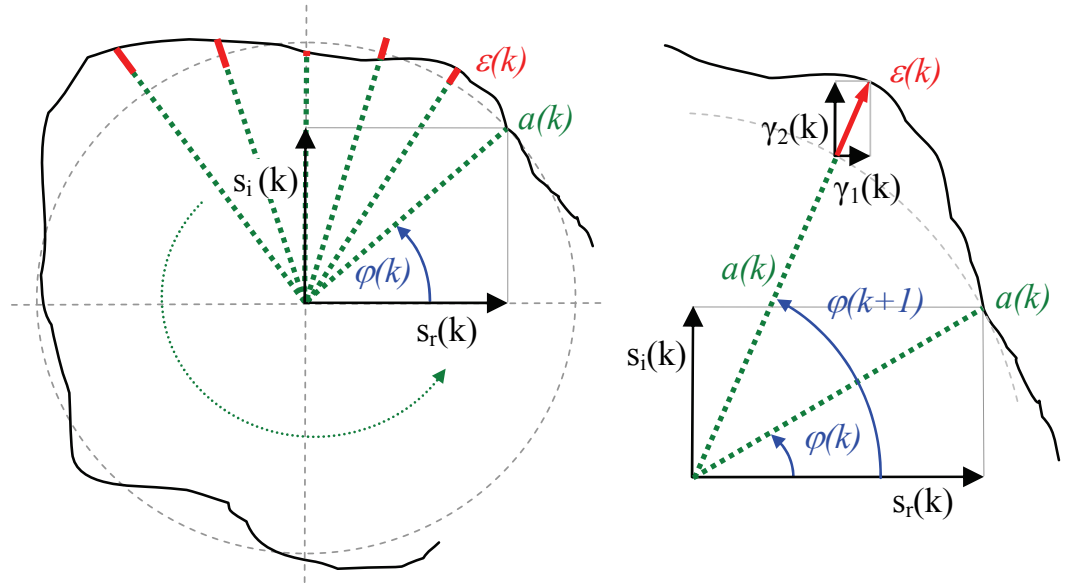

Fig. 1. The time changing phasor with its corresponding amplitude $a(k)$, phase $\varphi(k)$ and deviation $\varepsilon(k)$.

Equation (24) can be also rewritten in quadratic form (in vector domain, the transposed vectors and matrices are used)

$$
x^{T}(k+1) x(k+1)=(A \cdot x(k)+A \cdot \bar{\Gamma}(k) \cdot \xi(k))^{T} \cdot(A \cdot x(k)+A \cdot \bar{\Gamma}(k) \cdot \xi(k))
$$

The converted form of the equation

$$
\begin{aligned}
& x^{T}(k+1) \cdot x(k+1)=x(k)^{T} \cdot A^{T} \cdot A \cdot x(k)+x(k)^{T} \cdot A^{T} \cdot A \cdot \bar{\Gamma}(k) \cdot \xi(k)+ \\
& +\xi(k) \cdot \bar{\Gamma}(k)^{T} \cdot A^{T} \cdot A \cdot x(k)+\bar{\Gamma}(k)^{T} \cdot A^{T} \cdot A \cdot \bar{\Gamma}(k) \cdot \xi(k)^{2}
\end{aligned}
$$

The quadratic form equality is valid also for the recursive amplitude equation

$$
a(k+1)^{2}=(a(k)+\varepsilon(k))^{2}=a(k)^{2}+2 a(k) \varepsilon(k)+\varepsilon(k)^{2}
$$

Using (27) and the following knowledge of the state product

$$
a(k)^{2}=x^{T}(k) x(k)
$$

the equation (26) can be rewritten into (30) where product of system matrices $A^{T} \cdot A$ is substituted by the identity matrix $I$ as follows 


$$
A^{T} \cdot A=I \Rightarrow x(k)^{T} \cdot A^{T} \cdot A \cdot x(k)=x(k)^{T} \cdot x(k) ; \quad \bar{\Gamma}(k)^{T} \cdot A^{T} \cdot A \cdot \bar{\Gamma}(k)=\bar{\Gamma}(k)^{T} \cdot \bar{\Gamma}(k)
$$

The result is following

$$
\begin{aligned}
& \underbrace{x^{T}(k+1) \cdot x(k+1)}_{a(k+1)^{2}}= \\
& =\underbrace{x(k)^{T} \cdot x(k)}_{a(k)^{2}}+x(k)^{T} \cdot \bar{\Gamma}(k) \cdot \xi(k)+\xi(k) \cdot \bar{\Gamma}(k)^{T} \cdot x(k)+\bar{\Gamma}(k)^{T} \cdot \bar{\Gamma}(k) \cdot \xi(k)^{2}
\end{aligned}
$$

The main reason of this derivative description is the need to obtain a relation between $\varepsilon$ and $\bar{\Gamma}$. Application of mean value operator on (30) results in the equation where only the required variables are presented:

$$
\begin{aligned}
& E\left(a(k)^{2}\right)+\underbrace{E(2 a(k) \varepsilon(k))}_{0}+E\left(\varepsilon(k)^{2}\right)= \\
& =E\left(a(k)^{2}\right)+\underbrace{E\left(x(k)^{T} \cdot \bar{\Gamma}(k) \cdot \xi(k)\right)}_{0}+\underbrace{E\left(\xi(k) \cdot \bar{\Gamma}(k)^{T} \cdot x(k)\right)}_{0}+E\left(\bar{\Gamma}(k)^{T} \cdot \bar{\Gamma}(k) \cdot \xi(k)^{2}\right)
\end{aligned}
$$

and resulting equation which connects $\varepsilon$ and $\bar{\Gamma}$ is

$$
\sigma_{\varepsilon}^{2}=\bar{\Gamma}(k)^{T} \cdot \bar{\Gamma}(k)
$$

The $\bar{\Gamma}$ vector consists of two components (because of second order model) and the result from multiplying of the $\bar{\Gamma}$ vectors is the sum of the square vector components

$$
\bar{\Gamma}(k)=\left[\begin{array}{l}
\bar{\gamma}_{1}(k) \\
\bar{\gamma}_{2}(k)
\end{array}\right] \Rightarrow \bar{\Gamma}(k)^{T} \cdot \bar{\Gamma}(k)=\bar{\gamma}_{1}(k)^{2}+\bar{\gamma}_{2}(k)^{2}
$$

Using (32) the first condition for selection of $\bar{\Gamma}$ is obtained as

$$
\sigma_{\varepsilon}^{2}=\bar{\gamma}_{1}(k)^{2}+\bar{\gamma}_{2}(k)^{2} \text {. }
$$

The second condition can be obtained from the presumption that the phasor of the state $x(k)$ and of the deviation $\varepsilon(k)$ have the same normal line. Going from the estimation of the state components $x_{r}(k)$ and $x_{i}(k)$ the ratio between them should be the same as the ration between $\bar{\gamma}_{1}(k)$ and $\bar{\gamma}_{2}(k)$ (see(35)). To be precise, there is a product of vector $\bar{\Gamma}$ and stochastic variable $\xi$ in the ratio equation but because of the same stochastic value in sample $k$ the variable $\xi$ is then cancelled out from the ratio (therefore the $\gamma_{1}$ and $\gamma_{2}$ coordinates of $\varepsilon$ in Fig. 1. are also used without variable $\xi$ )

$$
\frac{\bar{\gamma}_{2}(k) \cdot \xi(k)}{\bar{\gamma}_{1}(k) \cdot \xi(k)}=\frac{x_{i}(k)}{x_{r}(k)}
$$

The converted form of the ratio equation

$$
\bar{\gamma}_{2}(k)=\frac{x_{i}(k)}{x_{r}(k)} \cdot \bar{\gamma}_{1}(k)
$$


is then substituted into (34) and results in formula for the first parameter of vector $\bar{\Gamma}(k)$

$$
\bar{\gamma}_{1}(k)=\sqrt{\frac{\sigma_{\varepsilon}{ }^{2} \cdot x_{r}(k)^{2}}{x_{r}(k)^{2}+x_{i}(k)^{2}}}
$$

and for the second one

$$
\bar{\gamma}_{2}(k)=\sqrt{\frac{\sigma_{\varepsilon}{ }^{2} \cdot x_{i}(k)^{2}}{x_{r}(k)^{2}+x_{i}(k)^{2}}}
$$

These equations rewritten for the original model definition with the vector $\Gamma(k)$ have the following form

$$
\Gamma(k)=\left[\begin{array}{l}
\gamma_{1}(k) \\
\gamma_{2}(k)
\end{array}\right]=A \cdot\left[\begin{array}{l}
x_{r}(k) \cdot \sqrt{\frac{\sigma_{\varepsilon}{ }^{2}}{x_{r}(k)^{2}+x_{i}(k)^{2}}} \\
x_{i}(k) \cdot \sqrt{\frac{\sigma_{\varepsilon}{ }^{2}}{x_{r}(k)^{2}+x_{i}(k)^{2}}}
\end{array}\right] .
$$

This formula describes the characteristics of the amplitude deviation between samples $k$ and $k+1$ namely in the state model in (18) which is then together with (19) used as a model for Kalman estimation. In Kalman filter computation of $\Gamma(k)$ an estimate $\mu(k)$ of state $x(k)$ is used (see(46)). Selection of value $\Delta$ in the output equation (20) is the next step of defining the state model. Output $y(k)$ of $n$ component model is formed only from the real parts of the components. This is acquired through the vector $C$ and the product of $C \cdot x(t)$. The value $\Delta$ makes it possible to include the measurement noise $(\Delta \cdot \eta(k))$ into the output equation. In fact, $\Delta$ is the variance of additive error of the measuring chain. For example in case where the sensor accuracy class is defined in statistical sense so that the absolute additive error is $95 \%$ quantile in limits of product of accuracy class $\times$ measuring range, the $95 \%$ quantile represents the range of $0 \pm 2 \sigma$ where $\sigma$ is the standard deviation of additive error. In this case the following form of the measurement error variance can be accepted

$$
\Delta=\left(\frac{\text { accuracy class } \times \text { measuring range }}{2}\right)^{2} .
$$

\section{Use of Kalman filter for estimation of the signal components}

In this work, an adaptive Kalman filter based approach is used for estimation of the analyzed signal. Signal is modelled as sum of resonators (signal components) and it is required that the estimated components are complex functions because of efficient computation of the instantaneous frequency.

\subsection{Discrete Kalman filter}

A discrete-time Kalman filter realizes a statistical estimation of the internal states of noisy linear system and it is able to reject uncorrelated measurement noise - a property shared by 
all Kalman filters. Let's assume a system with more components as mentioned above. Then the state matrix consists of following blocks:

$$
A_{n}=\left[\begin{array}{cc}
\cos \left(h \cdot \omega_{n}\right) & \sin \left(h \cdot \omega_{n}\right) \\
-\sin \left(h \cdot \omega_{n}\right) & \cos \left(h \cdot \omega_{n}\right)
\end{array}\right], .
$$

and the state noise vector blocks are defined as in (39). Then in state-variable representation, the description of the whole system, which is characterized by the sum of resonators, is given by the following matrices:

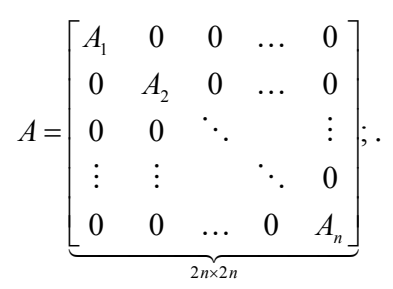

the output matrix is following

$$
C=\underbrace{\left[\begin{array}{lllllll}
1 & 0 & 1 & 0 & \ldots & 1 & 0
\end{array}\right]}_{1 \times 2 n} ;
$$

and state and measurement noise is characterized by the following parameters

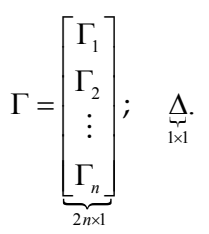

Commonly, the Kalman estimation includes two steps - prediction and correction phase. Let's assume that the state estimate $\mu(0)$ is known with an error variance $P(0)$. A priori value of the state at instant $k+1$ can be obtained as

$$
\mu(k+1)=A \cdot \mu(k)
$$

The measured value $y(k)$ is then used to update the state at instant $k$. The additive correction of the a priori estimated state at $k+1$ is according to (Vaseghi, 2000) proportional to the difference between the a priori output at instant $k$ defined as $C \cdot \mu(k)$ and the measured $y(k)$ :

$$
\mu(k+1)=A \cdot \mu(k)+K(k) \cdot(y(k)-(C \cdot \mu(k)))
$$

where $K(k)$ is the Kalman gain which guarantees the minimal variance of the error $x(k)-\mu(k)$.

Also, at each step the variance $P(k+1)$ of the error of $\mu(k+1)$ is calculated (see (Vaseghi, 2000)): 


$$
P(k+1)=A \cdot P(k) \cdot A^{T}+\Gamma \cdot \Gamma^{T}-K(k) \cdot\left(C \cdot P(k) \cdot A^{T}+\Delta \cdot \Gamma^{T}\right)
$$

This variance matrix is then used for calculation of Kalman gain in the next step of the recursive calculation (correction phase):

$$
K(k)=\left(A \cdot P(k) \cdot C^{T}+\Gamma \cdot \Delta^{T}\right) \cdot\left(C \cdot P(k) \cdot C^{T}+\Delta \cdot \Delta^{T}\right)^{-1}
$$

\subsection{Estimation of initial parameters}

The initial parameters for Kalman filter are obtained from the estimation of the signal spectrum. In principle, there are many ways how to fix the parameters, let's present two of them. Generally, there is need to define which modes, or more precisely which frequencies $\omega_{n}$, should be estimated and what is the variance of the amplitude on these frequencies.

The first way how to acquire the initial parameters is based on the short-time Fourier transform (STFT) algorithm. The half length of the STFT window can be used as an order of the modelled system (number of modelled resonators) and the corresponding frequencies are then the frequencies of the modelled signal components. The variation of the amplitude in the STFT frequency bands serves then as the estimation of the variation $\sigma_{\varepsilon}{ }^{2}$ of $\varepsilon$ for each resonator. This approach shows the Kalman filter method based on the short-time Fourier frequency analysis.

The second alternative is based on the estimation of spectral density by fitting an AR prediction model to the signal. The used estimation algorithm is known as Burg's method (Marple, 1987), which fits an AR linear prediction filter model of a specified order to the input signal by minimizing the arithmetic mean of the forward and backward prediction errors. The spectral density is then computed from the frequency response of the prediction filter. The AR filter parameters are constrained to satisfy the Levinson-Durbin recursion. The initial Kalman filter parameters (frequencies of the resonators) are then obtained as local maxima of estimated spectral density which are greater than a predefined level. These values indicate significant frequencies in spectral density and determine the order of the model.

The Fig. 2. shows the schema of the whole method. The estimated parameters from the input signal form the system model which is then used in the estimation phase of the algorithm.

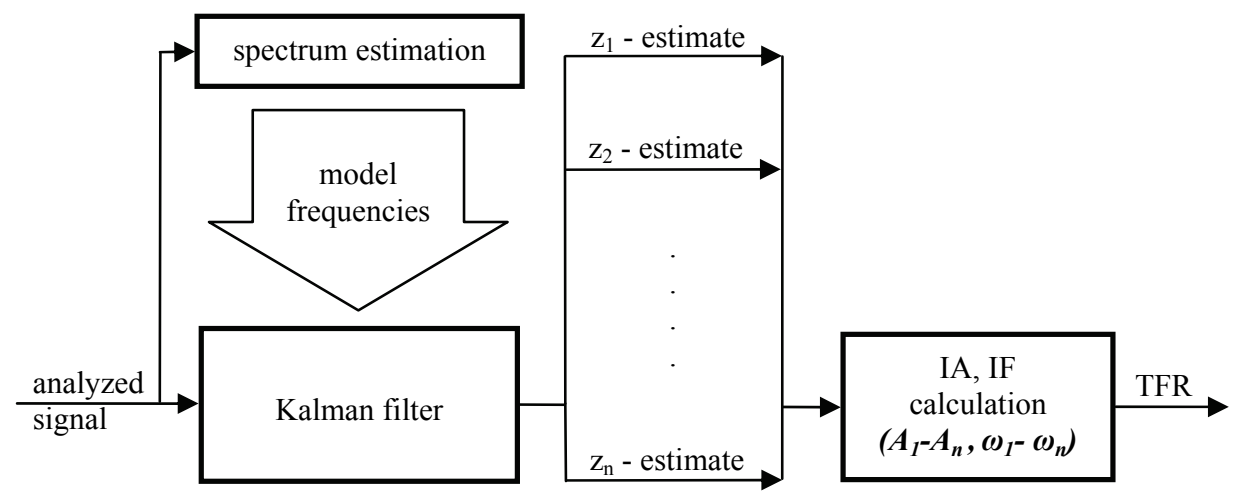

Fig. 2. Schema of the method for time-frequency decomposition of the signal using Kalman filter 
Kalman filter estimates the states of the modelled system in form of complex signal. It means that the imaginary parts of components are estimated simultaneously with the estimation of real signal components. This procedure substitute the Hilbert transform in generation of analytical signal and is performed recursively.

Each component in complex form serves then for calculation of the instantaneous amplitude and instantaneous frequency (see (6) and (8)). The output of the method is the timefrequency representation of the analyzed signal. The representation consists of amplitude curves with changing frequency in contrast to other time-frequency methods whose TF representation is computed in bands that is why the resolution in time and frequency is limited.

\section{Examples}

In this section, some examples of the time-frequency analysis using Kalman filter method are shown. The most important indicators in comparison with other analysis methods are the sharpness of the estimated frequency curves and the adaptability to the changes in the component amplitudes. These attributes are deciding for the next signal processing in the class of problems which are solved by authors.

Let's have a signal with three harmonic components, with $1 \mathrm{kHz}$ sampling rate and the total analyzed length of the signal is 1 second ( $N=1000$ points). Signal is formed by sinus functions with oscillation frequencies $f_{1}=10 \mathrm{~Hz}, f_{2}=30 \mathrm{~Hz}$ and $f_{3}=50 \mathrm{~Hz}$. The amplitude for all three components is set to 10 , the second component is set to zero for the first 0.5 seconds. The output noise with mean $m_{\eta}=0$ and variance $\sigma_{\eta}{ }^{2}=1$ was added to the simulated signal. The initial parameters for Kalman filter were obtained through Burg's AR linear prediction filter of order 10 and the level for local maxima in the Burg's spectral density was determined as $\max >1$. The initial conditions of Kalman estimator were set up in the following way: $\mu(0)=[1 \ldots 1]^{T}, \quad P(0)=10^{6} \cdot I, \Delta=1$, where $\operatorname{dim}(\mu)=n \times 1$ and $\operatorname{dim}(P)=2 n \times 2 n$.

The time-frequency representation of the instantaneous frequency and amplitude is shown in Fig. 3 (left). The result shows that the adaptation of one of the model component has an influence also on the instantaneous frequency estimates of other components which is expressed in the oscillation between 0.5 and 0.6 second (the overall oscillation of the instantaneous frequency is the effect of the output noise).

The adaptation rate is shown in Fig. 3 (right), where the comparison of estimate convergence of the component at $f_{2}=30 \mathrm{~Hz}$ using Hilbert approach and Kalman filter is performed. The Hilbert transform was applied artificially to the component itself, not to the analyzed signal (in order to compare the adaptation). The reason is the disadvantage of the Hilbert transform that requires the pre-processing of the signal through some signal decomposition method.

The above introduced Kalman filter method uses for decomposition of the signal into its components, as already mentioned, the model of sum of resonators and thus simultaneously decomposes the signal and estimates the time progression of its components. 

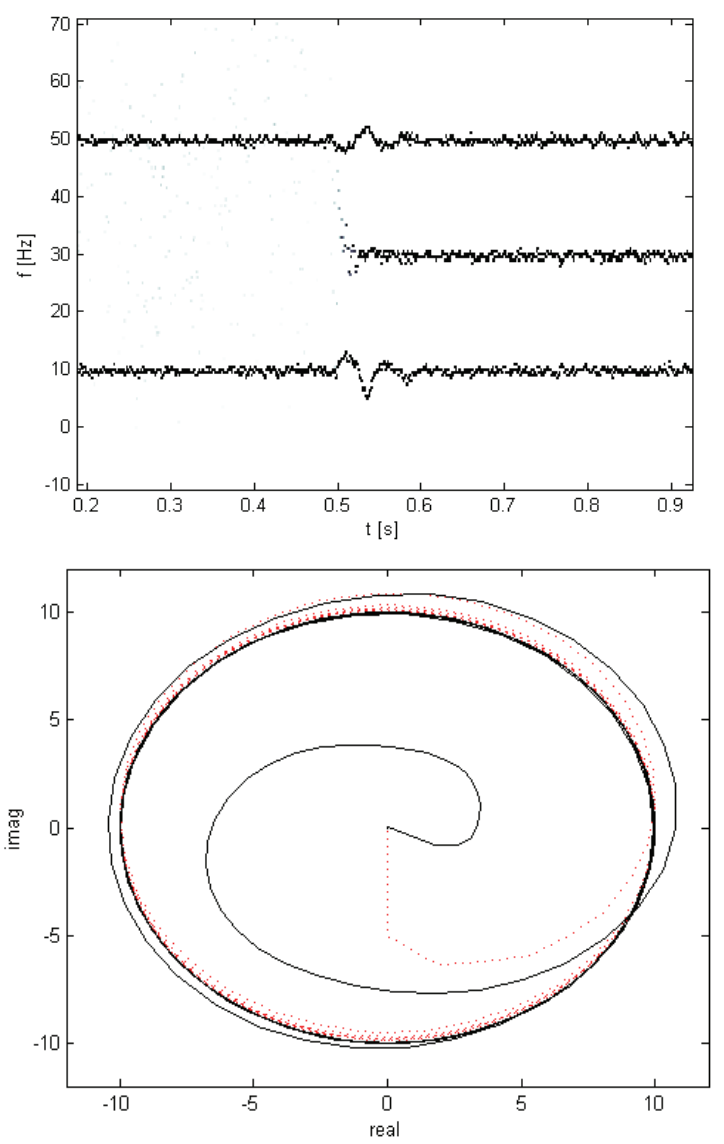

Fig. 3. Simulation signal with three components (left). Complex signal (right) obtained through Hilbert transform (dotted line) and through Kalman estimation (solid line).

The algorithm based on Kalman estimation is also illustrated on another signal example (see Fig. 4). It consists from signal components, where the first one is the stationary harmonic signal with constant frequency. This signal part is summed in time with the concave parabolic chirp signal where its frequency changes from 0.45 to 0.1 of normalized frequency. Both components exist in time between $t=100$ and $t=900$. The initial conditions of Kalman estimator were set up as mentioned in previous example and initial frequencies of the model were obtained through Burg's AR linear prediction filter of order 25 (number of estimated frequencies was $n=10$ and thus order of the estimated system was 20).

The Kalman filter method is compared with other typical time-frequency methods and the results are shown in Fig. 4. For comparison, the short-time Fourier transform - STFT, wavelet transform - WT (using Morlet wavelet) and smoothed pseudo Wigner-Ville distribution - SPWVD, were used. The output of Kalman estimation in time-frequency domain has comparable resolution as the output of the SPWVD. The other methods have relative wide frequency bands containing the signal energy. 

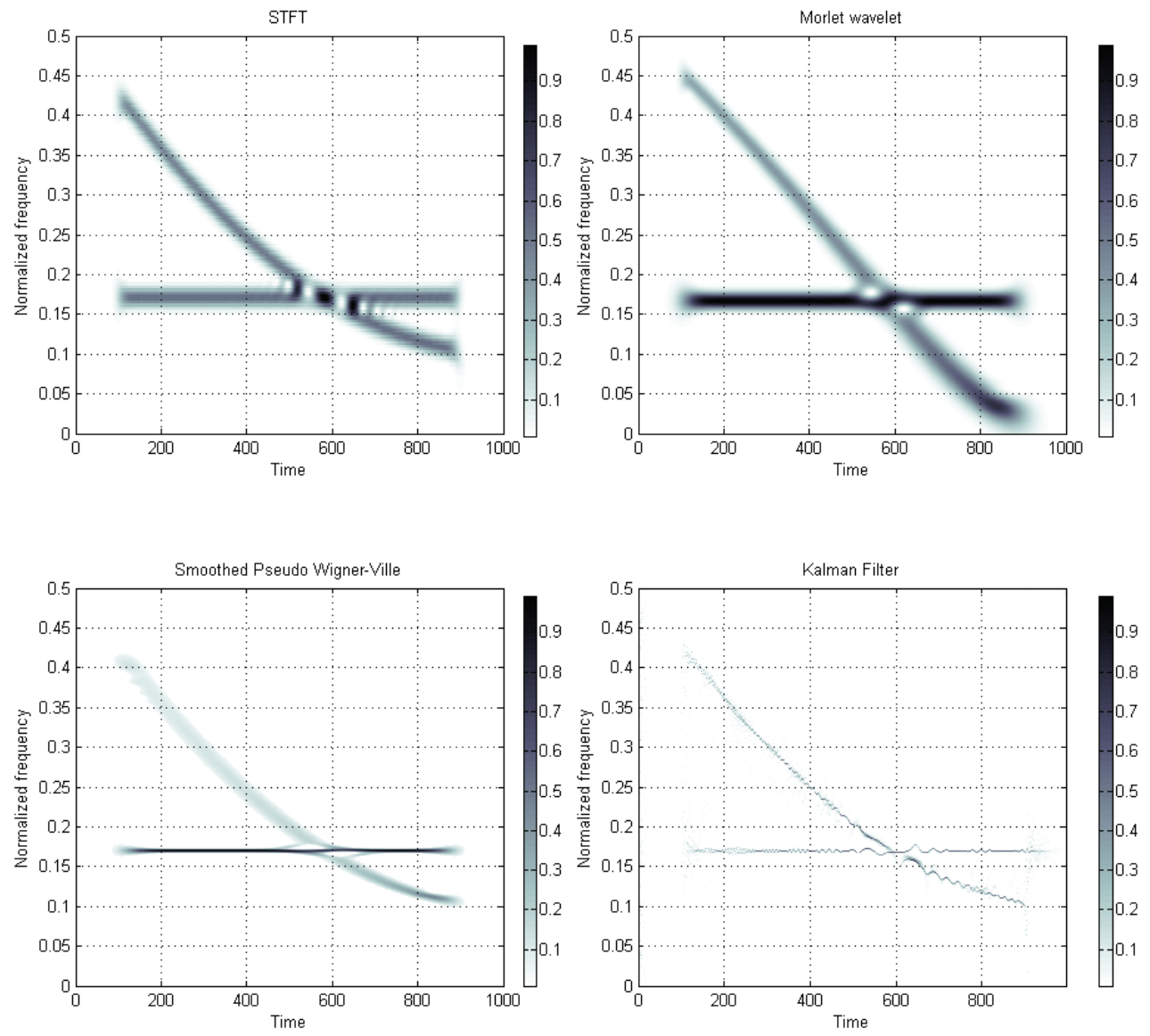

Fig. 4. Estimation of instantaneous frequency and amplitude of harmonic and chirp signal components.

Similarly, the result of methods comparison in Fig. 5. shows the Kalman filter method and SPWVD as more detailed time-frequency approaches. The test signal consists, in this case, of four harmonic components and the accuracy of the methods to identify the frequency and time of the component origin or end is tested. The signal begins again in time $t=100$ and ends in $t=900$. The frequency changes in $t=300$ and $t=600$, whereas there are two components simultaneously between these times. The ability of methods to distinguish between these two component frequencies is visible. SPWVD and Kalman filter representations display these two simultaneous components separately. On the contrary STFT and WT representations contain time-frequency artefacts between components and that's why the identifying of each component separately using these methods could be in such cases really difficult. 

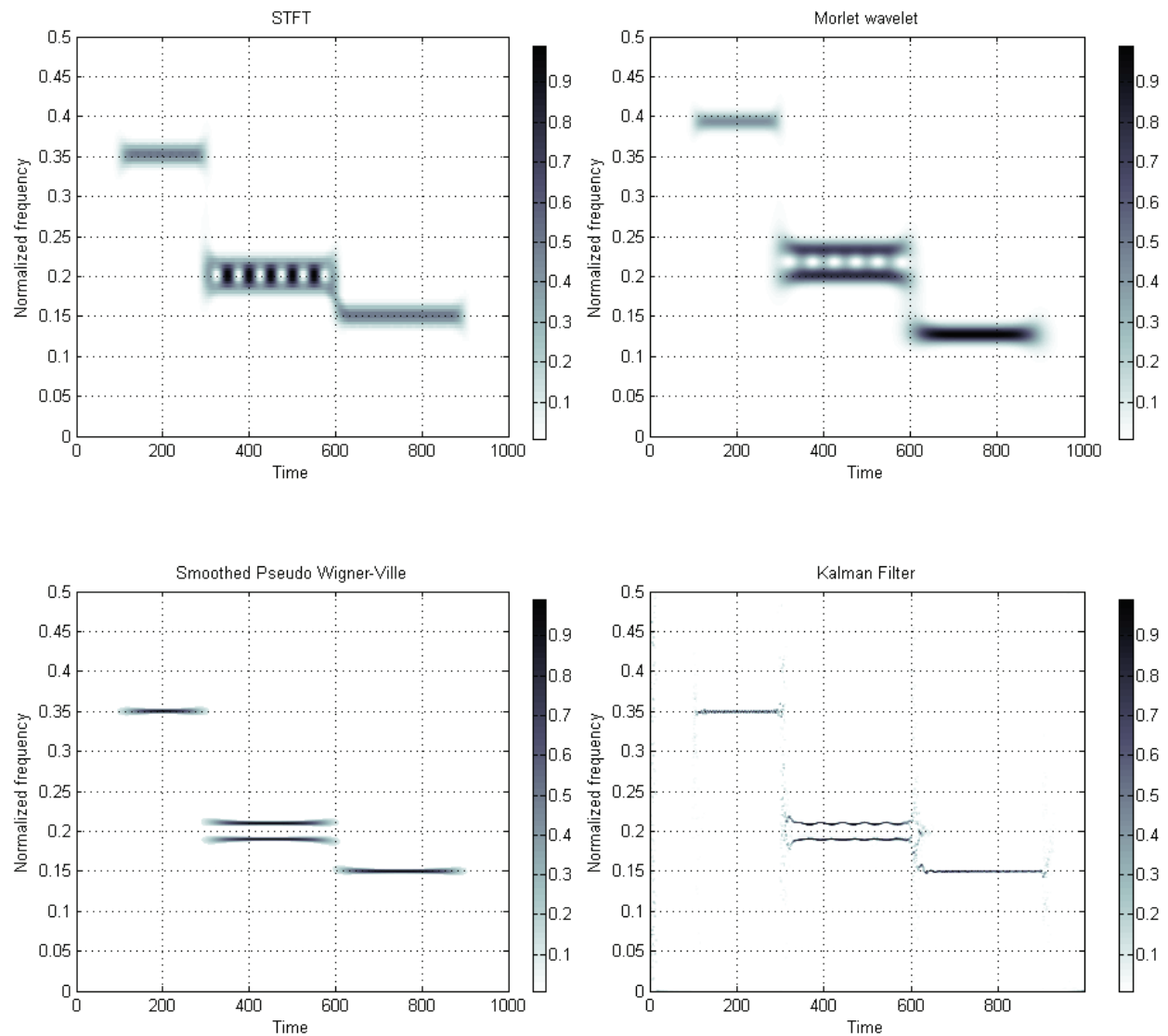

Fig. 5. Estimation of instantaneous frequency and amplitude of harmonic components of the signal.
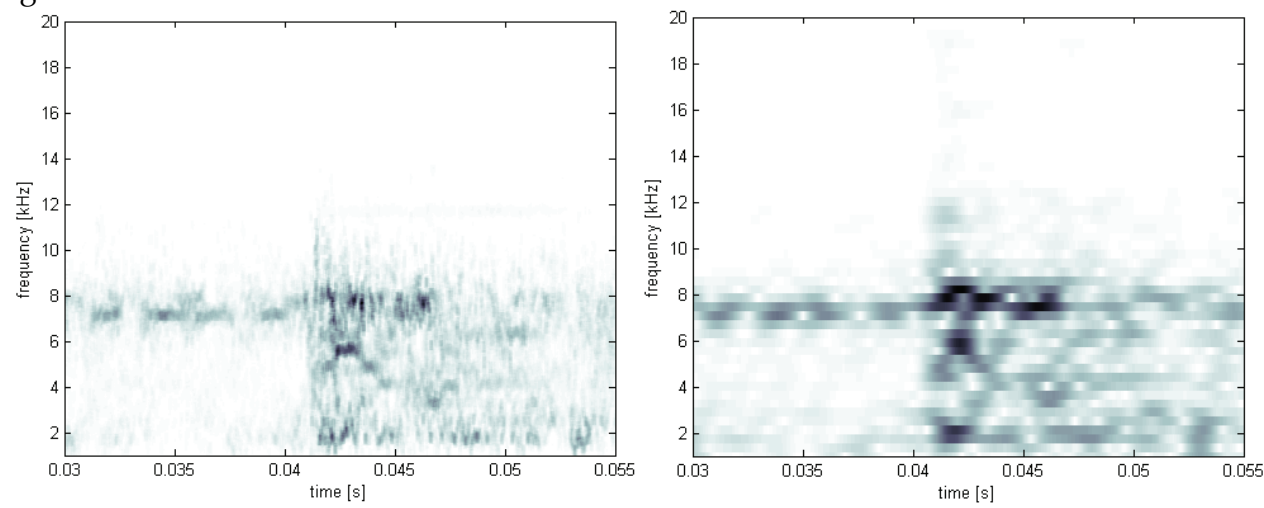

Fig. 6. Estimation of instantaneous frequency and amplitude of loosened part impact signal: Kalman filter method (left) and STFT (right). 
The last example is the transform of the acoustic signal from the real equipment where the nonstationary event took place. This event is measured result of loosed part impact in primary circuit of nuclear power plant. Signal was measured with $100 \mathrm{kHz}$ sampling rate. For comparison, in Fig. 6, the time-frequency-amplitude responses of STFT (right) and of the Kalman method (left) are compared. Kalman filter signal model was initialized with frequencies from the frequency analysis with window of length $2^{8}$ samples. The same window length with 75\% overlapping was used for analysis by means of STFT (Fig. 6 right). An event (impact) which occurs at time 0.041 seconds is well visible in both spectrograms (see the frequency band $1-12 \mathrm{kHz}$ ). The advantage of the Kalman version of spectrogram is its better resolution in time and frequency than in STFT spectrogram (which is used by authors for improvement of impact location).

\section{Conclusion and perspective}

The new approach for time-frequency signal analysis and for representation of instantaneous frequency and amplitude has been introduced in this chapter. The procedure is based on the Kalman estimation and shares its advantages regarding the suppression of measurement noise. In this method the Kalman filter serves for dissociation of signal into modes with well defined instantaneous frequency. The analyzed signal is modelled as sum of second-order subsystems (resonators) whereby the Kalman filter decompose the system by estimating these subsystems. Simultaneously with the signal decomposition the time progression of signal components in complex form is evaluated. This procedure utilizes the adaptive feature of the Kalman filter and it is done recursively for each sample.

In cases where the short-time Fourier transform cannot offer sufficient resolution in timefrequency domain, there can be taken the advantage of this method despite of higher computational severity. Also the experimental results show that the resolution of the introduced method is equal or higher than in other usual time-frequency techniques.

In vibro-diagnostic methods, where time-frequency information is used for location of nonstationary events, the sharpness of the introduced method is helpful for the improvement of the non-stationary event location. The next tasks of the Kalman filter method development are the systematization of the results from different actually solved problems in loose part monitoring and finding the new branches where the method could be effectively apllied.

\section{References}

Boashash, B. (1992a). Estimating and Interpreting the Instantaneous Frequency of a Signal Part 1: Fundamentals, Proceedings of the IEEE, Vol. 80, No. 4, pp. 520-538, April 1992, ISSN 0018-9219

Boashash, B. (1992b). Estimating and Interpreting the Instantaneous Frequency of a Signal Part 2: Algorithms and Applications, Proceedings of the IEEE, vol. 80, no. 4, pp. 540568, April 1992, ISSN 0018-9219

Cohen, L. (1995). Time Frequency Analysis: Theory and Applications, Prentice Hall PTR., ISBN 978-0135945322, New Jersey

Fairman, F.W. (1998). Linear Control Theory: The State Space Approach, John Wiley\& Sons, ISBN 0471974897, Toronto 
Hahn, S.L. (1996). Hilbert Transforms in Signal Processing, Artech House Publishers, ISBN 978089006886, Boston

Huang, N.E., et al. (1998). The empirical mode decomposition and the Hilbert spectrum for nonlinear and non-stationary time series analysis, Proceedings: Mathematical, Physical and Engineering Sciences, Vol. 454, No. 1971, pp. 903-995, March 1998, ISSN 1364-5021

Marple, S.L. (1987). Digital Spectral Analysis with Applications, Prentice Hall Signal Processing Series, Prentice Hall, Englewood Cliffs, ISBN 978-0132141499, New Jersey.

Vaseghi, S.V. (2000). Advanced Digital Signal Processing and Noise Reduction, John Wiley \& Sons Ltd, ISBN 0471626929, New York 


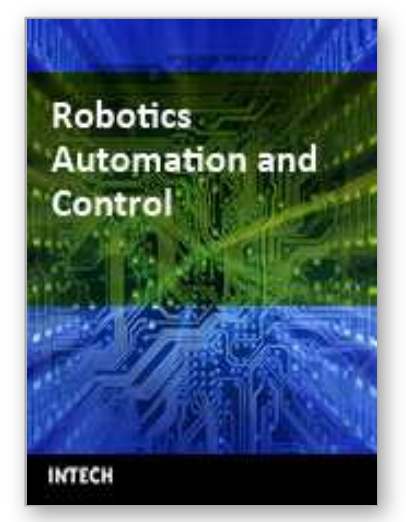

\author{
Robotics Automation and Control \\ Edited by Pavla Pecherkova, Miroslav Flidr and Jindrich Dunik
}

ISBN 978-953-7619-18-3

Hard cover, 494 pages

Publisher InTech

Published online 01, October, 2008

Published in print edition October, 2008

This book was conceived as a gathering place of new ideas from academia, industry, research and practice in the fields of robotics, automation and control. The aim of the book was to point out interactions among various fields of interests in spite of diversity and narrow specializations which prevail in the current research. The common denominator of all included chapters appears to be a synergy of various specializations. This synergy yields deeper understanding of the treated problems. Each new approach applied to a particular problem can enrich and inspire improvements of already established approaches to the problem.

\title{
How to reference
}

In order to correctly reference this scholarly work, feel free to copy and paste the following:

Jindrich Liska and Eduard Janecek (2008). Time-Frequency Representation of Signals Using Kalman Filter, Robotics Automation and Control, Pavla Pecherkova, Miroslav Flidr and Jindrich Dunik (Ed.), ISBN: 978-9537619-18-3, InTech, Available from: http://www.intechopen.com/books/robotics_automation_and_control/timefrequency_representation_of_signals_using_kalman_filter

\section{INTECH}

open science | open minds

\section{InTech Europe}

University Campus STeP Ri

Slavka Krautzeka 83/A

51000 Rijeka, Croatia

Phone: +385 (51) 770447

Fax: +385 (51) 686166

www.intechopen.com

\section{InTech China}

Unit 405, Office Block, Hotel Equatorial Shanghai

No.65, Yan An Road (West), Shanghai, 200040, China

中国上海市延安西路65号上海国际贵都大饭店办公楼 405 单元

Phone: +86-21-62489820

Fax: +86-21-62489821 
(C) 2008 The Author(s). Licensee IntechOpen. This chapter is distributed under the terms of the Creative Commons Attribution-NonCommercialShareAlike-3.0 License, which permits use, distribution and reproduction for non-commercial purposes, provided the original is properly cited and derivative works building on this content are distributed under the same license. 